\title{
Lactating dairy cows adapt quickly to being milked by an automatic milking system
}

\author{
J. A. Jacobs and J. M. Siegford ${ }^{1}$ \\ Department of Animal Science, Michigan State University, East Lansing 48824
}

\begin{abstract}
Transitioning a dairy herd to an automatic milking system (AMS) from a conventional parlor system may be stressful for the cow, as many changes occur during this process. Chronic stress may affect the welfare of the cow, and acute stress during milking can decrease milk yield. Therefore, it is important to quantify if and how long stress during adaptation to an AMS might persist. Seventy-seven cows with acceptable udder and teat conformation that would not interfere with adaptation to the AMS and that were lactating $\{\mathrm{n}=18$, early [0 to $100 \mathrm{~d}$ in milk (DIM)]; $\mathrm{n}=27$, mid (100 to 200 DIM); and $n=32$, late (200+ DIM) $\}$ for the full duration of the project were chosen for observation. All cows had been milked previously in a double- 6 herringbone milking parlor. Four stress-related behaviors [step-kick behavior both before and after attachment of teat cups, elimination (urination and defecation instances), and vocalization] were recorded during milking by trained observers, whereas milk yield was automatically recorded by the AMS. Data were collected for 24-h periods beginning on the day the cows transitioned to milking in the AMS (d 0), and on d 1, $2,4,8,16$, and 32 thereafter. Instances of elimination and vocalization were greater on $\mathrm{d} 0$ compared with all other days (elimination: $\mathrm{d} 0=3.1 \pm 0.09$, d $1=$ $0.6 \pm 0.07$, and $0 \pm 0$ instances thereafter; vocalization: $\mathrm{d} 0=1.7 \pm 0.07, \mathrm{~d} 1=0.05 \pm 0.04$, and $0 \pm 0$ instances thereafter). Milk yield increased between d 0 $(18.3 \pm 1.7 \mathrm{~kg})$ and $\mathrm{d} 1(30.9 \pm 1.7 \mathrm{~kg})$. Primiparous cows $(\mathrm{n}=28)$ were more likely than multiparous cows $(\mathrm{n}=49)$ to display step-kick behaviors both before $(8.3$ $\pm 2.5 ; 5.5 \pm 0.6$, respectively) and after (15.6 \pm 2.4 ; $13.3 \pm 1.3$, respectively) teat cup attachment during milking. Eight days after introducing the cows to the AMS, over $60 \%$ of the herd was milking voluntarily and $95 \%$ of the herd was milking voluntarily within a month, which suggests that cows did not find the AMS aversive. Greater elimination and vocalization behavior
\end{abstract}

Received July 8, 2011.

Accepted October 27, 2011.

${ }^{1}$ Corresponding author: siegford@msu.edu and lower milk yield on d 0 relative to subsequent days indicated initial stress and discomfort with the milking process in the new system; however, the cows appeared to adapt within $24 \mathrm{~h}$.

Key words: dairy cow, robotic milking, adaptation rate, stress-related behavior

\section{INTRODUCTION}

Automatic milking systems (AMS) were first introduced commercially in the Netherlands in 1992, and have become popular throughout Europe. Over the course of the last decade, AMS have been adopted by some farms in North America. Currently, about 8,000 farms worldwide are using an AMS, with $90 \%$ of the world's AMS farms located in northwestern Europe (de Koning, 2010). Of the remaining 10\% of AMS farms, about $90 \%$ are located in Ontario and Quebec (Rodenburg and House, 2007). Perhaps due to the differences in average herd size, the high initial cost of acquiring 1 or more AMS, farmer perceptions with regard to technology, low profit margins for farms, or the relatively low cost of labor; the United States largely has yet to adopt the AMS as an alternative to the traditional milking parlor. With this in mind, research aimed at analyzing the ease (or struggle) of the transition between milking with a conventional parlor and milking with an AMS might be beneficial to the industry.

Several studies have compared discomfort and stress behaviors between conventional parlors and AMS (Hopster et al., 2002; Wenzel et al., 2003; Gygax et al., 2008) with conflicting results. Most conventional parlors are designed to have multiple cows milking simultaneously and adjacent to each other. The majority of AMS are single-stall milking units and, thus, cows are milked in social isolation, separate from their herd mates. Cows milked in social isolation in unfamiliar surroundings show signs of acute stress including decreased milk yield, presumably due to reduced oxytocin secretion (Rushen et al., 2001). In addition, these surroundings have been suggested to increase the incidences of defecation, urination, and vocalization in dairy cows (Rushen et al., 1999). The AMS uses technology to milk cows independent of human assistance. Human 
interaction decreased stress and fear responses (Rushen et al., 2001). Thus, for cows familiar with being milked in the company of other cows and humans, the change to robotic milking might be stressful.

Another major change for cows adapting to the AMS is a different motivation to enter the milking stall. Cows are no longer driven to be milked in a large group 2 or 3 times daily. Instead, they are expected to voluntarily go to the AMS of their own accord, and as individuals. For this reason, the AMS needs to be viewed as an attractive, rewarding experience, to ensure the cows will return. In most AMS systems, a highly palatable concentrate is offered in the milking stall to entice a visit. But, if the milking process or environmental changes are stressful for the cow, the addition of a concentrate reward may not be enough to encourage regular visits to the AMS. Cows that do not visit the AMS on their own accord must be physically fetched to the AMS, which increases labor time and cost for the farmer. For this reason, it becomes particularly important to quantify the length of time required for transitioning cows to adapt to milking in the new system.

Although it is important to measure stress responses during the period between transitioning from a conventional parlor to an AMS, the process of collecting physiological indicators of stress may in itself act as a stressor (Weiss et al., 2004), confounding the responses to the new milking systems. Therefore, observational techniques were used to compare responses to the AMS over time. Vocalization, defecation, and urination incidences are considered indicators of acute stress or fear in cattle (de Passillé et al., 1995; Grandin, 1998); and increased movement (stepping and kicking) is considered a sign of agitation (Grandin, 1993). The objective was to determine the duration of time required for cows to adapt to milking in an AMS by measuring behaviors associated with the stress response. It was hypothesized that the cows would initially exhibit high levels of stress-related behaviors, and these would gradually decrease over time as the cows habituated to the AMS environment and milking process.

\section{MATERIALS AND METHODS}

Prior to the start of the study, all protocols were submitted to and approved by the Michigan State University Institutional Animal Care and Use Committee.

\section{Animals and Husbandry}

Eighty-eight lactating Holstein dairy cows made the transition from a freestall barn with a double- 6 herringbone parlor to a new freestall barn housing 2 Lely Astronaut A3 Automatic Milking Systems (Lely
Holding S.à r.l., Maassluis, the Netherlands) on July 7, 2009. Prior to the transition, cows were milked 3 times daily in the parlor and were managed in 3 groups: 2 -yr olds, multiparous cows, and special needs cows (i.e., cows with clinical lameness or reproductive challenges). Once moved to the new facility, all cows from previous groups were divided into 2 groups balanced for parity and stage of lactation, with each group having access to a single AMS. Seventy-seven cows were selected for inclusion in the current study based on acceptable teat and udder conformation, and the expectation that they would be lactating for the duration of the project (group 1: $\mathrm{n}=38$; group 2: $\mathrm{n}=39$ ). Cows were in their first to sixth lactation with $207 \pm 15.2$ DIM. Except for the handedness of the milking system (i.e., group 1 had a left-handed milker and group 2 a right-handed milker, meaning the robotic arm approached the cow from either the left side or right side, respectively), the type of AMS, feeding alley, and stall layouts were identical for both groups. Feeding and management practices were identical for both groups. Cows remained in their assigned groups throughout the study.

For ease of visual identification, cows were marked with individual numbers ranging from 0 to 76 over their rib cages and thurls using either bleaching product (L'Oréal Quick Blue; L'Oréal, Paris, France) for black areas or black hair dye (L'Oréal Starry Night; L'Oréal) for white areas. The cows assigned had never been milked by an AMS before; however, all had been previously milked in the conventional parlor, and had general experience with machine milking. The daily milk yield in the double- 6 herringbone parlor before the transition was $29.1 \pm 0.76 \mathrm{~kg}$. Free cow traffic was allowed in the new barn with the exception of $\mathrm{d} 0$ and during limited periods on $\mathrm{d} 1$ and 2 , when cows were driven to the AMS in accordance with a protocol developed by farm staff and Lely representatives to train cows to milk in the AMS (Figures 1 and 2). The AMS visits were positively reinforced by feeding concentrate pellets in the milking stall at each successful visit. Concentrate amount offered in the AMS varied depending upon DIM and expected milk yield. The time of day feeding occurred remained identical between the old and new barns (0500 and $1500 \mathrm{~h}$ ), but TMR composition was adjusted slightly in the new barn to allow for the inclusion of concentrate pellets that were being fed in the AMS.

\section{Experimental Procedure}

Cows transitioned to the new barn on the morning of July 7, 2009 (d 0) following a transition protocol devised in consultation with Lely representatives (Figures 1 and 2 ). Cows were milked at $0400 \mathrm{~h}$ in the conventional par- 
lor before being moved into the new barn on $\mathrm{d} 0$. Cows were manually driven to their respective AMS on $\mathrm{d} 0$ between 0800 and $0759 \mathrm{~h}$ the next day. The first milking of each cow in the AMS involved farm personnel assisting the process by helping guide the robotic arm to the udder, as well as performing manual teat cleaning. On d 2, free cow traffic was allowed, and voluntary milking was expected. Milking intervals for each cow were programmed into the AMS depending upon stage of lactation and expected milk yield. Starting on d 2, cows that were overdue for milking were driven (i.e., fetched) to the AMS every $12 \mathrm{~h}$, at 0400 and $1600 \mathrm{~h}$ each day. In subsequent sections, cows needing to be driven to the AMS after d 8 are described as fetched cows.

To measure adaptation rates of the cows being milked by the new AMS, trained data collectors observed the cows once they entered the milking stall of the AMS for each successful milking event. Observations began with each cow's first milking (d 0 at $0800 \mathrm{~h}$ ) by the new robots, and then were conducted on d 1, 2, 4, 8, 16, and 32 thereafter. Data were collected for 24 -h periods on each observation day (i.e., from 0800 until 0759 the next day) with observers seated $2.43 \mathrm{~m}$ from each milking unit in identical chairs. In accordance with Martin and Bateson (1993), behavior was recorded continuously with focal animal sampling each time an individual accessed the milking stall. Observers recorded the number of steps and kicks (Gygax et al., 2008) both before and after teat cups were attached, as well as instances of urination, defecation, and vocalizations. Milk yield at each milking was recorded automatically by the AMS. The identity of the cow was recorded each time it had to be fetched to the AMS after the eighth day of milking in the new system.

\section{Lely Start-up Procedure (2009)}

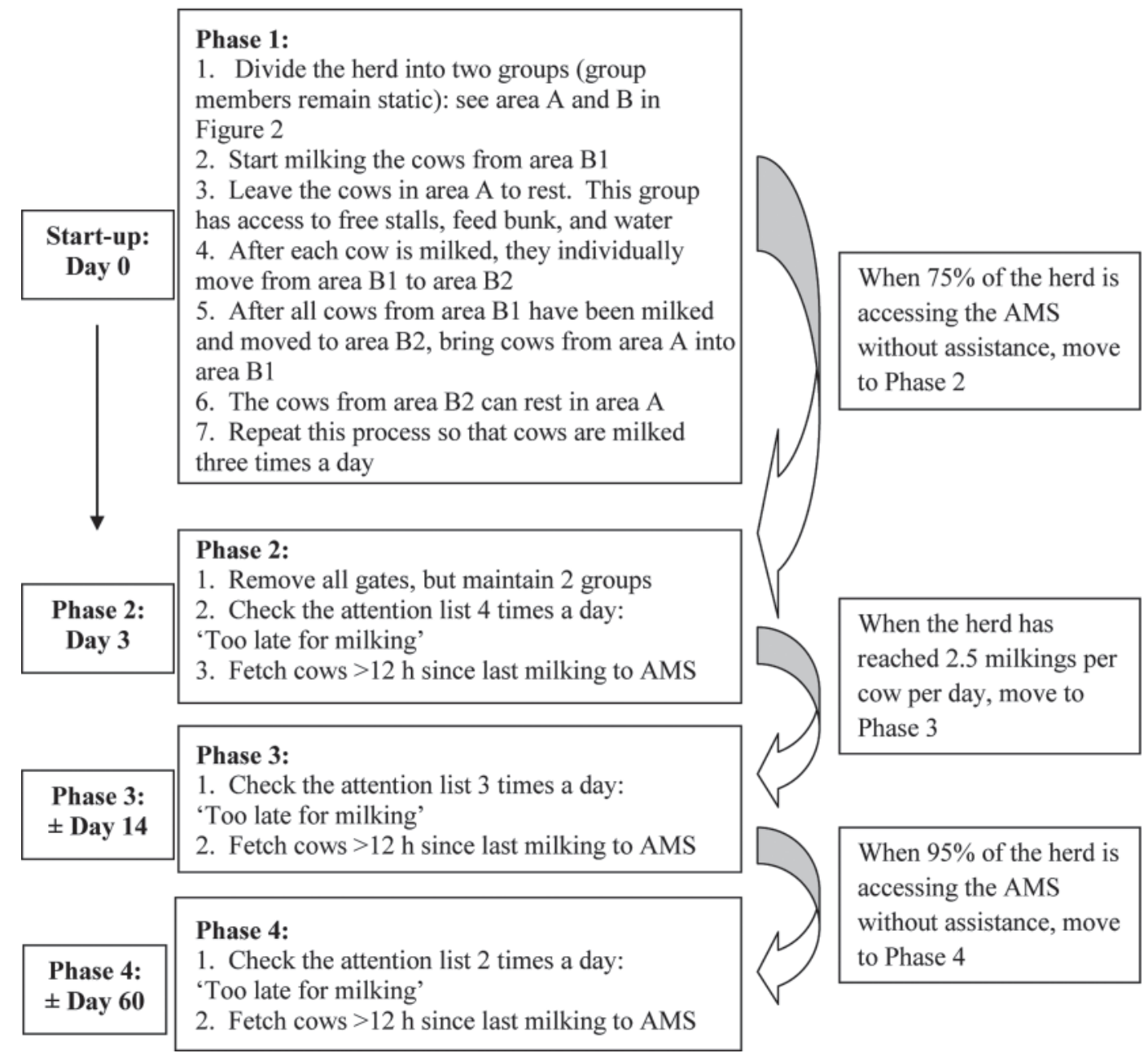

Figure 1. This procedure was adapted from the Lely start-up procedure 2009 (Lely Holding S.à r.l., Maassluis, the Netherlands). Goals to reach specific phases were approximate. AMS = automatic milking system. 


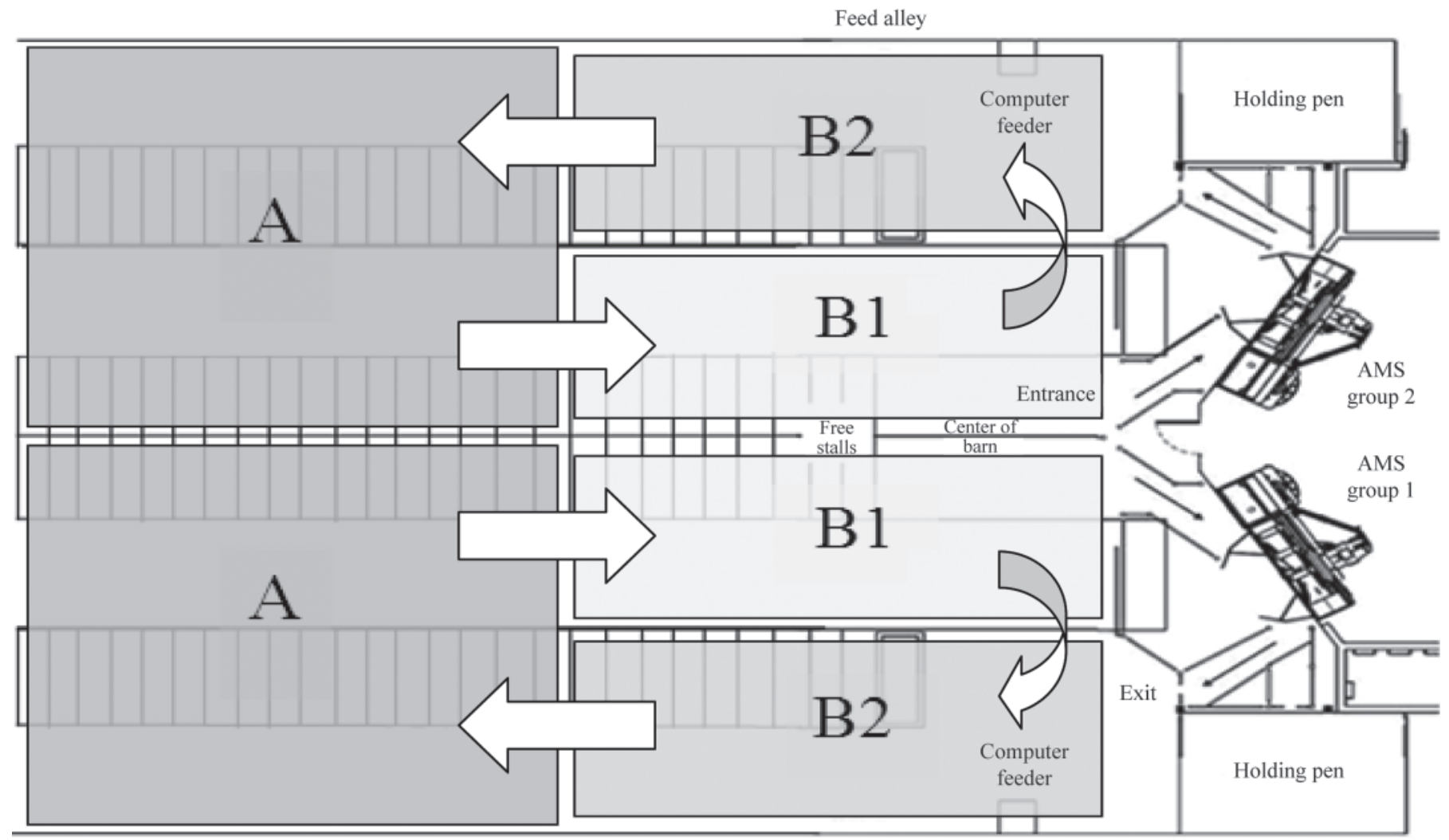

Feed alley

Figure 2. Temporary gates split each group within the herd into 2 main areas (A and B) for training during the start-up procedure on d 0 to 3. Cows were moved from area B1 for milking in the automatic milking system (AMS), and moved to area B2 after milking. Cows in area A were at rest. Once all cows had been milked and moved to area B2, they were moved into area A. Cows previously in area A were moved into area B1 for milking. This was repeated 3 times daily to achieve 3 milkings per day, except on d 0 when 2 milkings were carried out.

\section{Statistical Analysis}

Artifacts. During the study, 1 cow's data were removed from the data set as she managed to move herself from group 2 to group 1 on $\mathrm{d} 3$. Because the AMS differ for the 2 groups in robotic arm orientation, her adaptation rates may have been affected, making it necessary to eliminate her data from the records.

Stress-Related Behaviors. Data were analyzed with a mixed model procedure (PROC MIXED, SAS 9.1.3; SAS Institute Inc., Cary, NC) to compare the effect of day, parity, and DIM on stress-related behaviors (steps and kicks before and after teat attachment, vocalization, urination and defecation instances) and milk yield using the following model:

$$
\begin{aligned}
Y_{i j k l}= & \mu+G_{i}+D_{j}+G_{i} \times D_{j}+P_{k}+L_{l}+P_{k} \\
& \times L_{l}+P_{k} \times D_{j}+L_{l} \times D_{j}+e_{i j k l},
\end{aligned}
$$

where $Y_{i j k l}=$ milk yield or stress-related behavior of interest, $\mu=$ overall mean, $G_{i}=$ fixed effect of group ( $i=2$ groups), $D_{j}=$ repeated measures of day $(j=1$ to $7 \mathrm{~d}$ ), $P_{k}=$ parity ( $k=2$ parity levels), $L_{l}=$ stage of lactation $\left(l=1\right.$ to 3 levels), and $e_{i j k l}=$ residual error.

Data for 76 cows were evaluated. Only data from a cow's first milking event on each observation day were analyzed, with the intention of controlling for the time of day that the milking events occurred. Results were considered statistically significant at a probability of $\alpha$ less than 0.05 . The 3 -way interaction between parity (primiparous or multiparous), stage of lactation (early, mid, or late), and day was evaluated, but removed from the model when not significant $(P>0.05)$. Statistical differences were based on differences between least squares means of day for the following comparisons: vocalization, elimination (urination and defecation instances were grouped together), and milk yield. For steps and kicks both before and after teat attachment to the milking cups, data were log-transformed to meet assumptions of normality. Data in graphs are presented using untransformed least squares means estimates. Error bars represent the standard errors of the means.

Milk Yield Before and After Transition. For statistical evaluation of milk yield differences before 
and after the transition to the new milking system, the paired $t$-test procedure was used (PROC TTEST, SAS 9.1; SAS Inst. Inc.). Milk yield data (0800 to 0759 $\mathrm{h}$ each day) from 48 cows were analyzed. The remaining 29 cows for which behavioral adaptation data were collected had incomplete milk yield data (i.e., July 3 milk yield data were unavailable), and these cows were removed from the milk yield analysis. The milk yields of cows milked on July 3, 2009 ( -4 d before transition) in the conventional milking parlor and cows milked on July 11, 2009 ( $+4 \mathrm{~d}$ after transition) in the AMS were compared using a paired $t$-test. Results were considered significant at $P<0.05$. The following model was used to assess milk yield:

$$
Y_{i}=\mu+D_{i}+e_{i}
$$

where $Y_{i}=$ milk yield, $\mu=$ overall mean, $D_{i}=$ day $[i$ $=\mathrm{d} 1(-4 \mathrm{~d}$ before transition $), \mathrm{d} 2(+4 \mathrm{~d}$ after transition)], and $e_{i}=$ residual error.

Fetching. For statistical evaluation of the relationship between day of study and the number of cows fetched to the AMS a correlation analysis was performed to calculate the Pearson coefficient of correlation (PROC CORR, SAS version 9.1; SAS Inst. Inc.). Data for all 88 cows being milked, including cows that were deemed unsuitable based on teat and udder confirmation, were included. If a cow was fetched more than 1 time per day, she was only included once for that day. Results were indicated as statistically significant at a probability of $\alpha$ less than 0.05 . Descriptive statistics (mean \pm SEM, range, and percent of unsuitable cows of total cows fetched) were performed to depict the fetched rate of unsuitable cows and of cows deemed suitable for inclusion in this adaptation study.

\section{RESULTS}

\section{Step-Kick Before Teat Cup Attachment}

Primiparous cows $(8.3 \pm 2.5)$ were more likely than multiparous cows $(5.5 \pm 0.6)$ to display stepping and kicking behaviors before teat cup attachment $(P<$ 0.05 ; Figure 3$)$. A trend was seen in the overall effect of day $(P=0.09)$, with the number of steps and kicks before attachment decreasing after d 0 .

\section{Step-Kick After Teat Cup Attachment}

Primiparous cows $(15.6 \pm 2.4)$ were more likely than multiparous cows $(13.3 \pm 1.3)$ to display step and kick behaviors after teat cup attachment (Figure 4; $P<$ $0.05)$. A trend was seen for the overall effect of day $(P$ $=0.06)$, with the number of steps and kicks after teat cup attachment increasing to $\mathrm{d} 4$.

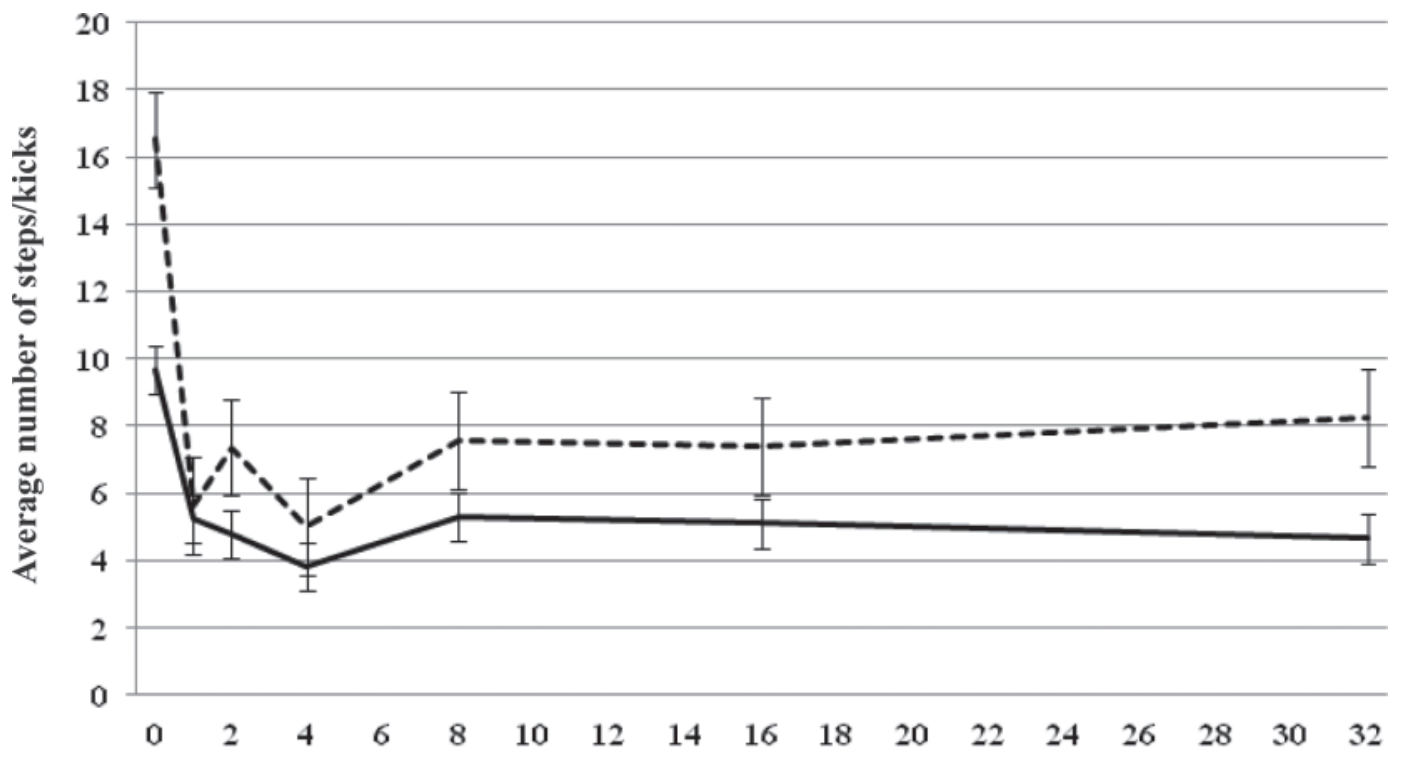

Days after transition

Figure 3. Throughout the study, primiparous cows $(\mathrm{n}=27)$ displayed more step-kick behaviors before teat cup attachment $(P<0.05)$ than did multiparous cows $(\mathrm{n}=49)$. A tendency was observed for steps and kicks before teat attachment to decrease over time $(P=0.09)$. The data represent least squares means of untransformed data \pm standard error of the means. The dashed line represents primiparous cows, whereas the solid line represents multiparous cows. 


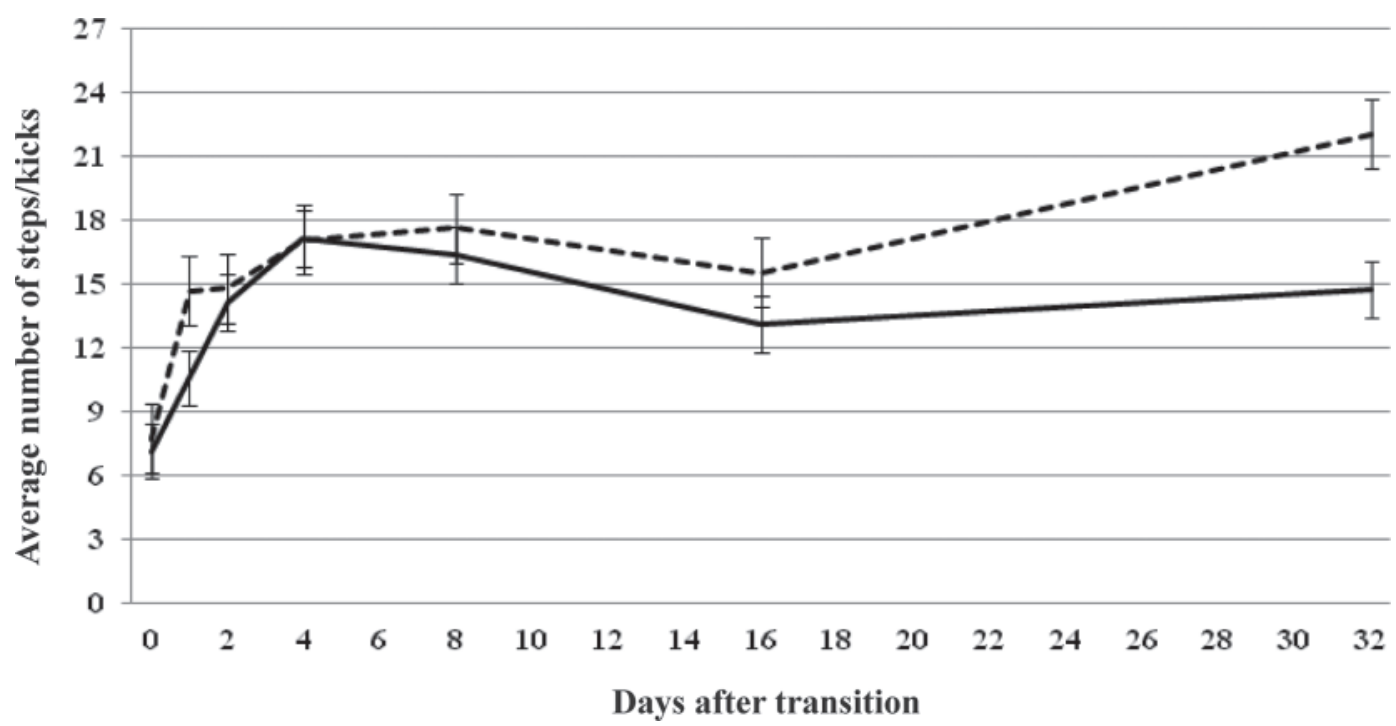

Figure 4. Throughout the study, primiparous cows $(\mathrm{n}=27)$ displayed more step-kick behaviors after teat attachment and during milking $(P$ $<0.05)$ than did multiparous cows $(\mathrm{n}=49)$. A tendency was observed for steps and kicks before teat cup attachment to increase over time $(P$ $=0.06)$. The data represent least squares means of untransformed data \pm standard error of the means. The dashed line represents primiparous cows, whereas the solid line represents multiparous cows.

\section{Elimination and Vocalization Instances}

Elimination instances decreased between d 0 (3.1 \pm $0.09)$ and $d 1(0.6 \pm 0.07 ; P<0.001)$ and remained at 0 instances for the remainder of the study (Figure 5). Similarly, vocalization instances decreased between d $0(1.7 \pm 0.07)$ and d $1(0.5 \pm 0.04 ; P<0.001)$, and remained at 0 instances for the remainder of the study (Figure 5). No effects of parity or DIM were observed for either factor.

\section{Milk Yield}

Day, parity, and DIM affected milk yield $(P<0.05)$. Milk yield on d 0 was lower than all other days (Figure 6 ; d $0=18.3 \pm 1.7 \mathrm{~kg}, \mathrm{~d} 1=30.9 \pm 1.7 \mathrm{~kg} ; P<0.05)$. In general, multiparous cows $(33.1 \pm 1.4 \mathrm{~kg})$ had higher milk yields compared with primiparous cows (26.4 \pm $1.5 \mathrm{~kg} ; P<0.05)$. In addition, cows in early lactation $(33.9 \pm 1.6 \mathrm{~kg})$ had higher milk yields compared with cows in mid $(30.2 \pm 1.6 \mathrm{~kg})$ and late lactation (25.1

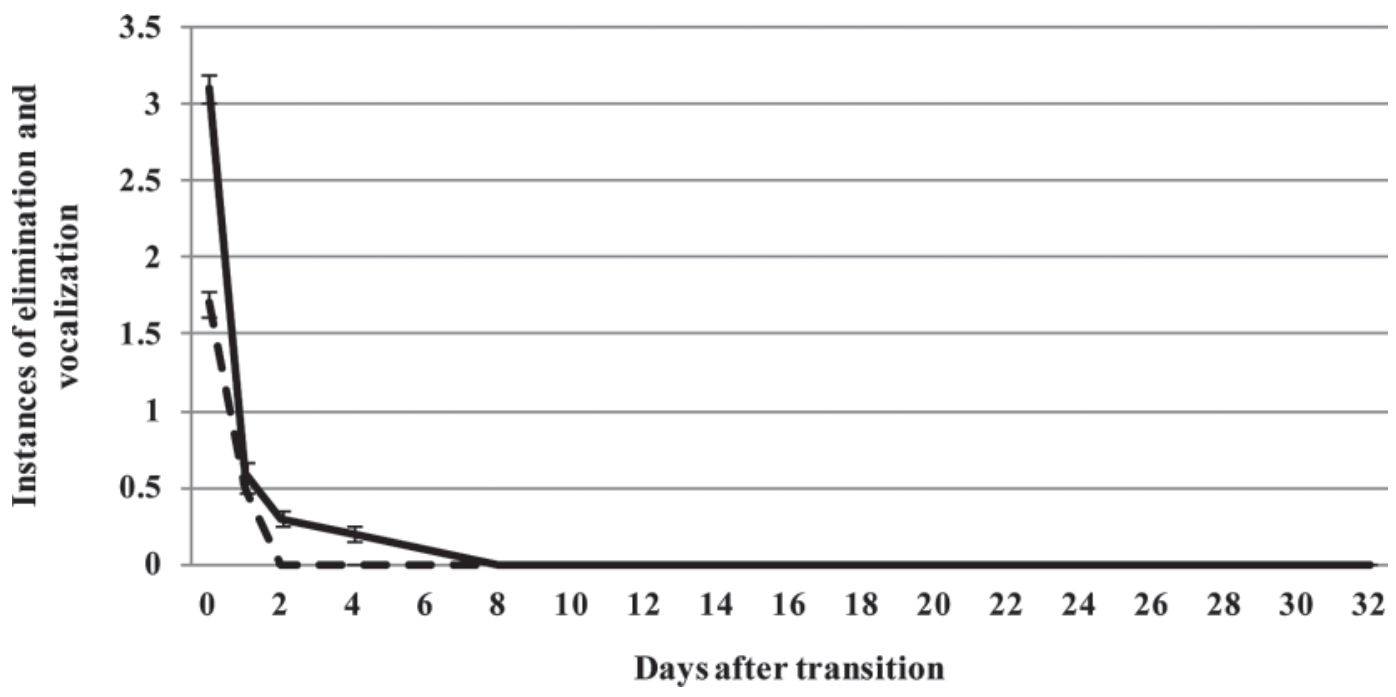

Figure 5. Instances of both elimination and vocalization behaviors decreased over time $(P<0.001)$. The dashed line represents elimination behaviors, whereas the solid line represents vocalization behaviors. 
Table 1. Descriptive data on milking performance of dairy cows transitioning from a conventional milking system to an automatic milking system during the experimental period of $32 \mathrm{~d}(\mathrm{n}=88)$

\begin{tabular}{lccccc}
\hline $\begin{array}{l}\text { Day of } \\
\text { collection }\end{array}$ & $\begin{array}{c}\text { Total } \\
\text { visits }\end{array}$ & $\begin{array}{c}\text { Total } \\
\text { milkings }\end{array}$ & $\begin{array}{c}\text { Total } \\
\text { refused visits }\end{array}$ & $\begin{array}{c}\text { Average } \\
\text { milkings (d/cow) }\end{array}$ & $\begin{array}{c}\text { Average milk } \\
\text { yield (kg) }\end{array}$ \\
\hline 0 & 191 & 164 & 5 & 2.1 & 15.0 \\
1 & 337 & 262 & 41 & 3.2 & 31.3 \\
2 & 354 & 277 & 55 & 3.3 & 33.1 \\
4 & 308 & 197 & 95 & 2.4 & 33.1 \\
8 & 340 & 223 & 101 & 2.7 & 34.6 \\
16 & 444 & 253 & 187 & 3.1 & 34.7 \\
32 & 386 & 260 & 119 & 3.0 & \\
\hline
\end{tabular}

$\pm 1.5 \mathrm{~kg} ; P<0.05)$. Table 1 provides a summary of milking performance of the cows during the $32 \mathrm{~d}$ experimental period.

\section{Milk Yield at $\pm 4 d$ from Transition}

Milk yield $(\mathrm{kg}) 4 \mathrm{~d}$ after the transition $(31.03 \pm 0.77$ $\mathrm{kg}$ ) to an AMS exceeded the average attained $4 \mathrm{~d}$ before transitioning to an AMS $(29.15 \pm 0.76 \mathrm{~kg} ; P<0.001)$.

\section{Fetched Cows}

Data on the number of cows that had to be fetched to milk was recorded beginning on $\mathrm{d} 8$ until $\mathrm{d} 32$. The relationship between the number of cows fetched and the date was linear and negatively correlated $(\mathrm{r}=$ $-0.73 ; P<0.001 ;$ Figure 7$)$. Cows initially deemed unsuitable for inclusion in this adaptation study $(\mathrm{n}=$ 11) needed to be fetched for milking more often (7.3 \pm 1.62 times) during $\mathrm{d} 8$ to 32 than the suitable cows $(\mathrm{n}=77 ; 3.28 \pm 0.53$ times $)$. The range of individual fetch counts for cows in either group was similar (0 to 17 times for unsuitable cows, 0 to 18 times for suitable cows). Unsuitable cows were responsible for $22.5 \%$ of all fetches from d 8 to d 32 despite representing only $12.5 \%$ of the herd.

\section{DISCUSSION}

Novel environments have been associated with increased stress levels in cattle (Grandin, 1997). When cows are moved from a conventional parlor system to an AMS they are exposed to a novel milking environment, and previous knowledge about acclimation rates during the transition to an AMS have been limited (Weiss et al., 2004). The current study may provide interested farmers with realistic expectations for their cows during the transition period from conventional parlors to

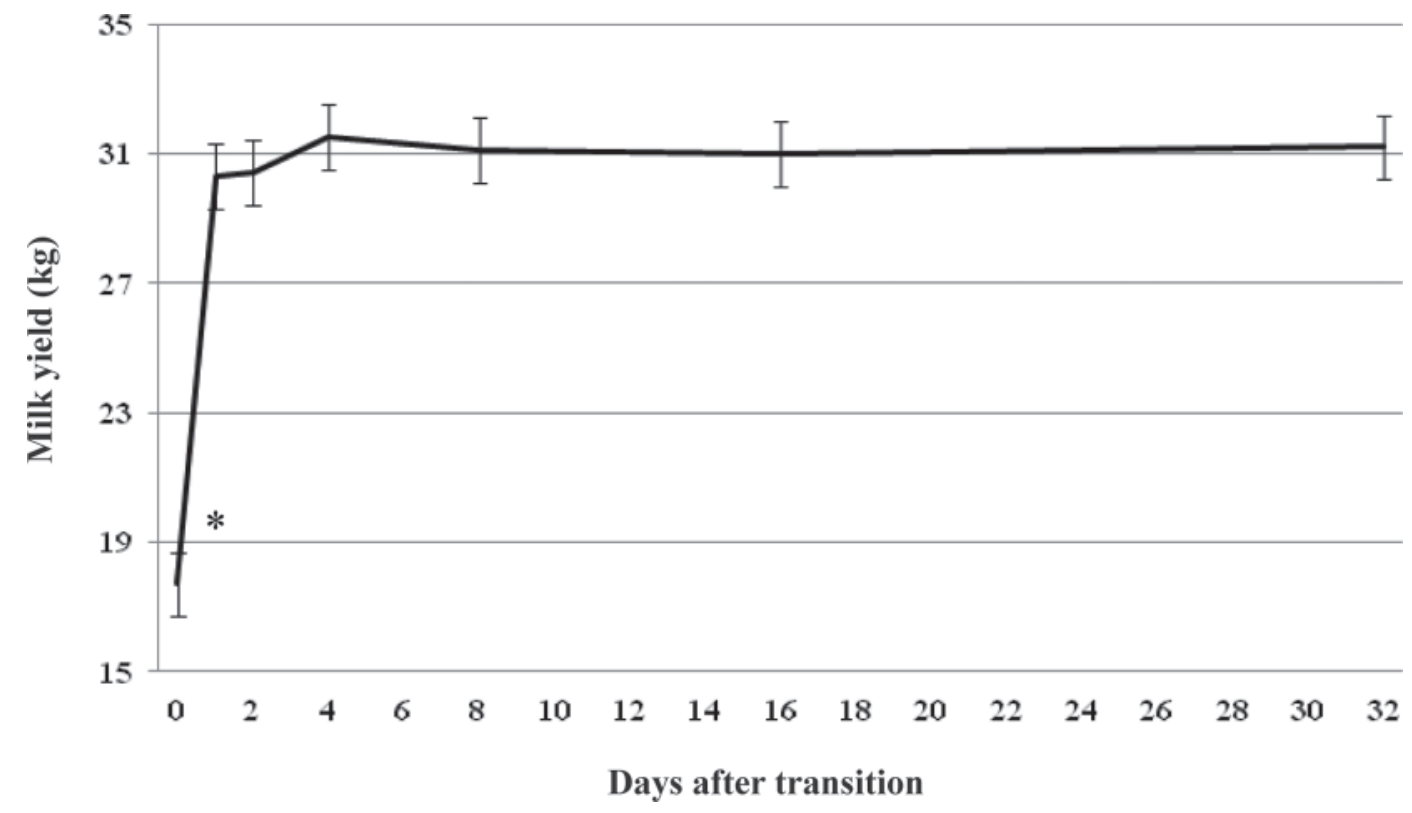

Figure 6. The effect of day on milk yield $(\mathrm{kg})$ was significant $(P<0.05)$. The presence of an asterisk indicates a difference from all other days $(P<0.05)$. The data represent means \pm standard error of the means. 


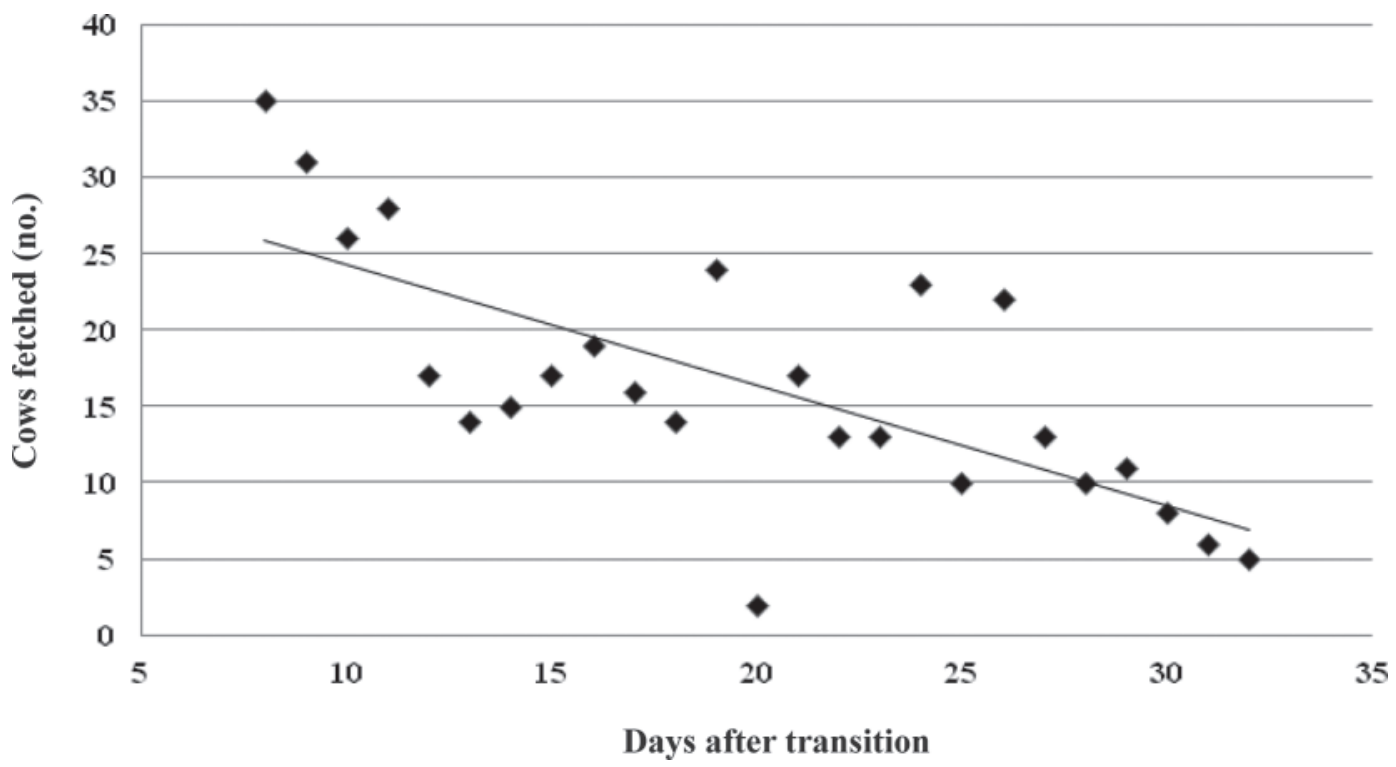

Figure 7. Relationship between the date $(\mathrm{x})$ and the number of fetched cows $(\mathrm{y}): \mathrm{y}=-0.67+0.13 \mathrm{x} ; \mathrm{r}=-0.73(P<0.001)$. July $15=\mathrm{d}$ 8; August $8=\mathrm{d} 32$.

AMS; and perhaps more importantly, ensure that cows are not chronically stressed following the transition, as this can lead to inferior health and welfare (Trevisi et al., 2006). It should be noted that the transition protocol used (Figures 1 and 2) may not be similar to those recommended by other manufacturers of AMS; therefore, these results may not be applicable to differing transition protocols.

During the first visit to the AMS (d 0), cows frequently vocalized, eliminated, stepped, and kicked before teat cup attachment, suggesting discomfort or stress with the novel milking environment. These behaviors may have been underestimated throughout, as cows deemed unsuitable for inclusion may have had a stronger response and adapted less quickly. Vocalization, defecation, and urination incidences are indicators of acute stress or fear in cattle (de Passillé et al., 1995; Grandin, 1998). Furthermore, increased movement (stepping and kicking) by cattle is considered as a sign of agitation (Grandin, 1993). The high incidences of vocalization, elimination, stepping, and kicking on d 0 suggest discomfort or stress during the initial milking event. The AMS was a completely novel milking unit for the cows, and novelty has been demonstrated to be a potent stressor for cattle (Grandin, 1997). This may be one of the reasons why stress and discomfort behaviors were present during the first day. In less than $24 \mathrm{~h}$, stepping and kicking before teat attachment decreased and vocalizing and eliminating in the AMS stall almost disappeared. The rapid decrease of these stress-related behaviors could be due to the cows becoming more comfortable with the milking stall, barn environment, and robotic milking equipment and process. It is important to note that the effect of group was not significant, indicating no differences in adaptation rates between the 2 groups, despite having access to differently facing AMS (left- vs. right-handed milking systems).

Primiparous cows exhibited greater step and kick responses compared with multiparous cows. It is possible that the step and kick response observed in the primiparous cows was exacerbated by their smaller stature. They had more room to move throughout the milking stall compared with the majority of the multiparous cows, and may have taken advantage of their size. Jago and Kerrisk (2011) observed a similar phenomenon in their primiparous cows.

Importantly, milk yield, which had decreased from an average of $29 \mathrm{~kg}$ per cow to an average of $16 \mathrm{~kg}$ per cow in the first $24 \mathrm{~h}$ in the new barn, rebounded to almost $32 \mathrm{~kg} /$ cow per day within $4 \mathrm{~d}$. These results support those of Weiss et al. (2004), who reported an initial decrease in milk yield during the first few AMS milking events when transitioning from a conventional parlor. Furthermore, cows achieved only 2.1 milking events on d 0, compared with approximately 3 milkings on all other days of data collection (Table 1). This may have been a contributing factor to the lower milk yield recorded on d 0; however, milk yield on a per milking basis is still lower for $\mathrm{d} 0$ than on subsequent days (d $0=7.14 \mathrm{~kg} /$ milking vs. $\mathrm{d} 1=9.78 \mathrm{~kg} /$ milking). The unfamiliar environment, coupled with a change in the milking process, may be the reason for the decrease 
in milk yield seen on d 0 . Regardless, by $\mathrm{d} 1$, the milk yield had returned to $29 \mathrm{~kg}$, indicating that the cows had become comfortable with the new milking process and stalls.

Another indication that the cows adapted quickly to the AMS were the number of cows voluntarily milking. Within a week of introducing the cows to the robotic milkers, over $60 \%$ of the herd was milking voluntarily. After 2 wk, over $75 \%$ of the herd was milking voluntarily and after $1 \mathrm{mo}, 95 \%$ of the herd was milking voluntarily. In terms of labor, this meant that only 5 cows needed fetching every $12 \mathrm{~h}$ for milking, whereas the remainder of the herd went through the robotic milkers voluntarily over 2.5 times per day. The voluntary milking rates were slightly lower than those described by Weiss et al., (2004), who reported a $97 \%$ voluntary rate 2 wk after transitioning to an AMS. Rodenburg and House (2007) reported large variation in voluntary milking between 43 surveyed herds. Five herds at the top of the scale averaged $97.5 \%$ of cows voluntarily milking, whereas the 5 herds at the bottom averaged $58.4 \%$. No obvious reason for such variation was provided, but perhaps in the future, an adaptable protocol and list of recommendations may be available for farmers considering making the switch to robotic milking to maximize the number of voluntary milking events exhibited by each transitioning herd.

The majority of farmers transitioning to an AMS will have to contend with several unsuitable cows (i.e., cows with imperfect teat and udder conformation, making teat attachment difficulty for the robotic arm) in their herd. In this study, 11 such cows were identified and excluded from the main focus of the study. However, an increased need to fetch these cows was observed (Figure 7 ), and all were culled or sold animals within 6 mo of transitioning to the robot. This evidence suggests need for future research aimed at understanding possible differences adaptation rates and increased labor and culling costs associated with unsuitable cows.

\section{CONCLUSIONS}

In summary, the cows adapted quickly to being milked by the AMS. One puzzling piece was the increase over time in the number of steps and kicks following teat attachment during the milking process. It is important to further investigate the rate of adaptation from a conventional parlor system to an AMS. It will be important for similar experiments to be completed (with the inclusion of unsuitable cows) to determine if these results are consistent with findings from other farms.

\section{ACKNOWLEDGMENTS}

Funding support for this research was generously provided by Michigan State University AgBioResearch (East Lansing) to Janice Siegford. Additional support was received from a grant to Michigan State University from the W. K. Kellogg Foundation (Battle Creek, MI) to establish a pasture-based dairy program at the Kellogg Biological Station (KBS, Hickory Corners, MI). We thank Rob Ashley and the KBS dairy barn staff for their assistance with this project. We gratefully acknowledge the statistical expertise of Nora Bello, Wei Wang, and Katya Ananyeva (all of Statistical Consulting Center, Michigan State University, East Lansing) for their assistance in the planning and analysis of this project. We also thank Courtney Daigle, Chelsey Shively, Krista Beeker, Aislin Hardee (all of Michigan State University, East Lansing), and Jolene Talaski and Mat Haan (Michigan State University, East Hickory Corners, MI) for their help collecting data. Special thanks to Dave Beede (Michigan State University, East Lansing), Marcia Endres (University of Minnesota, St. Paul), and Karen Plaut and Michael Schutz (both of Purdue University, West Lafayette) for their thoughtful comments and suggestions concerning the manuscript.

\section{REFERENCES}

de Koning, K. 2010. Automatic milking-Common practice on dairy farms. Pages 52-67 in Proc. 1st N. Am. Conf. Precision Dairy Mgmt. and 2nd N. Am. Conf. Robotic Milking, Toronto, Canada. Wageningen Academic Publishers, Wageningen, the Netherlands.

de Passillé, A. M., J. Rushen, and F. Martin. 1995. Interpreting the behavior of calves in an open-field test: A factor analysis. Appl. Anim. Behav. Sci. 45:201-213. doi:10.1016/0168-1591(95)00622Y.

Grandin, T. 1993. Behavioral agitation during handling is persistent over time. Appl. Anim. Behav. Sci. 36:1-9. doi:10.1016/01681591(93)90094-6.

Grandin, T. 1997. Assessment of stress during handling and transport. J. Anim. Sci. 75:249-257.

Grandin, T. 1998. The feasibility of using vocalization scoring as an indicator of poor welfare during cattle slaughter. Appl. Anim. Behav. Sci. 56:121-128. doi:10.1016/S0168-1591(97)00102-0.

Gygax, L., I. Neuffer, C. Kaufmann, R. Hauser, and B. Wechsler. 2008. Restlessness behaviour, heart rate and heart-rate variability of dairy cows milked in two types of automatic milking systems and auto-tandem milking parlours. Appl. Anim. Behav. Sci. 109:167-179. doi:10.1016/j.applanim.2007.03.010.

Hopster, H., R. M. Bruckmaier, J. T. N. Van der Werf, S. M. Korte, J. Macuhova, G. Korte-Bouws, and C. G. van Reenen. 2002. Stress responses during milking; comparing conventional and automatic milking in primiparous dairy cows. J. Dairy Sci. 85:3206-3216. doi:10.3168/jds.S0022-0302(02)74409-3.

Jago, J., and K. Kerrisk. 2011. Training methods for introducing cows to a pasture-based automatic milking system. Appl. Anim. Behav. Sci. 131:79-85. doi:10.1016/j.applanim.2011.02.002.

Martin, P., and P. Bateson. 1993. Measuring Behaviour. An Introductory Guide. 2nd ed. Cambridge University Press, Cambridge, UK.

Rodenburg, J., and H. K. House. 2007. Field observations on barn layout and design for robotic milking of dairy cows. In Proc. 6th 
Intl. Dairy Housing Conf., Minneapolis, MN. ASABE Publication Number 701P0507e (electronic only). Am. Soc. Agric. Biol. Eng., St. Joseph, MI.

Rushen, J., A. M. de Passillé, and L. Munksgaard. 1999. Fear of people by cows and effects on milk yield, behavior, and heart rate at milking. J. Dairy Sci. 82:720-727.

Rushen, J., L. Munksgaard, P. G. Marnet, and A. M. DePassillé. 2001. Human contact and the effects of acute stress on cows at milking. Appl. Anim. Behav. Sci. 73:1-14. doi:10.1016/S01681591(01)00105-8.

Trevisi, E., M. Bionaz, F. Piccioli-Cappelli, and G. Bertoni. 2006. The management of intensive dairy farms can be improved for better welfare and milk yield. Livest. Sci. 103:231-236. doi:10.1016/j. livsci.2006.05.009.

Weiss, D., S. Helmreich, E. Möstl, A. Dzidic, and R. M. Bruckmaier. 2004. Coping capacity of dairy cows during the change from conventional to automatic milking. J. Anim. Sci. 82:563-570.

Wenzel, C., S. Schönreiter-Fischer, and J. Unshelm. 2003. Studies on step-kick behavior and stress of cows during milking in an automatic milking system. Livest. Prod. Sci. 83:237-246. doi:10.1016/ S0301-6226(03)00109-X 